\title{
Scaling up from individual behaviour of Orius sauteri foraging on Thrips palmi to its daily functional response
}

\author{
Lia Hemerik • Eizi Yano
}

Received: 5 August 2010/Accepted: 20 April 2011/Published online: 13 May 2011

(C) The Author(s) 2011. This article is published with open access at Springerlink.com

\begin{abstract}
Functional responses of predators are generally measured under laboratory conditions at rather high prey densities. This is also true for the predation capability of the anthocorid predatory bug Orius sauteri (Poppius). To quantify the daily impact of one female Orius predator on its prey Thrips palmi Karny on greenhouse eggplants where the prey is present below the economic threshold density, we use its patch-leaving and feeding behaviour on eggplant leaves with different prey numbers and scale up to the larger spatio-temporal scale of the greenhouse and one foraging day by means of a simulation model. For this, we also use literature data on the distribution of $T$. palmi over eggplant leaves. The simulation results in a typical type II functional response for $O$. sauteri as a function of average T. palmi density: $O$. sauteri can find and eat approximately 10 prey items per day if $T$. palmi is present around its economic injury level. The daily mean number of prey eaten per $O$. sauteri predator, i.e., its predation capability, is highly sensitive to the actual baseline leaving tendency, the effect size of the presence of prey on the baseline leaving tendency and the effect size of the encounter rate with prey thereon.
\end{abstract}

Keywords Holling type II functional response .

Simulation · Survival analysis

\footnotetext{
L. Hemerik ( $\square)$

Biometris, Department of Mathematical and Statistical Methods, Wageningen University, P.O. Box 100, 6700 AC Wageningen,

The Netherlands

e-mail: lia.hemerik@wur.nl

E. Yano

Entomological Laboratory, Faculty of Agriculture, Kinki University, 3327-204 Nakamachi, Nara 631-8505, Japan
}

\section{Introduction}

Functional response is defined as the temporal rate at which an individual predator kills prey, namely the average number of prey eaten per individual predator per unit of time as a function of prey density. In models, the functional response is either assumed to be (1) linear, increasing with increasing prey density (Holling 1959; type I functional response), (2) reaching a plateau after a linear increase with increasing prey density (Holling 1959; type II functional response) or (3) increasing after an initial slow start up, levelling off to a plateau with increasing prey density (Holling 1959; type III functional response with sigmoid shape). The basis of Holling's disc equation (type II) is that the time budget of a predator is divided between searching for prey and handling prey (chasing, eating and digesting it). In this formulation, the search activity of the predator is constant irrespective of the density of prey. For the sigmoid functional response the predator intensifies its searching activity when prey densities increase.

The predacious anthocorid $O$. sauteri is well known to be one of the important indigenous natural enemies of T. palmi on eggplants in the field in Japan (Yano 1996). If field populations of Orius spp. including O. sauteri are conserved by using selective pesticides that do not affect Orius spp., then Orius populations suppress outbreak of T. palmi on eggplants in the field (Nagai 1993; Takemoto and Ohno 1996; Ohno and Takemoto 1997). Orius sauteri has also been demonstrated to suppress the population of T. palmi around the economic injury level on eggplants in greenhouses (Kawai 1995). Orius sauteri was registered in Japan in 1998 as a biopesticide of T. palmi and Frankliniella occidentalis (Pergande) in greenhouses.

The effectiveness of $O$. sauteri as a biological control agent of T. palmi mainly depends on its reproductive 
capacity and its predation capability. The intrinsic growth rate is generally used to indicate the reproductive capacity (Birch 1948). For T. palmi without predators around, this relative growth rate amounts to 0.102 per day (Kawai 1986), while that of $O$. sauteri fed with T. palmi is 0.128 per day (Nagai and Yano 1999). The reproductive capacities of these two species show no large differences. Therefore, the effectiveness of $O$. sauteri is mainly expected to depend on its predation capability. In this study, its functional response has been used to measure its predation capability.

The functional response of single Orius predators of different species are investigated frequently (e.g., McCaffrey and Horsburgh 1986; Isenhour et al. 1989, 1990; Coll and Ridgway 1995; Nagai and Yano 2000; Gitonga et al. 2002). As the experimental arena in most of the functional response studies in the laboratory were very small homogeneous spaces such as a Petri dish or a plastic vial to which the Orius individual was confined (McCaffrey and Horsburgh 1986; Isenhour et al. 1989, 1990; Coll and Ridgway 1995; Nagai and Yano 2000; Gitonga et al. 2002), the number of prey attacked in a given time period was over-estimated. In addition, the predator experienced higher prey densities than actual prey densities in greenhouses or in fields, where prey occur at densities around the economic injury level. In the experiments of Nagai and Yano (2000), the lowest density of thrips that could be used was three individuals per leaf, which is about five times higher than the economic injury level (of 0.55 per leaf). From such experiments it is impossible to directly extrapolate and obtain a reliable estimate of the predation capability of one Orius individual during one foraging day in a greenhouse where prey density is around the economic injury level. An estimate for this daily predation capability can be obtained in two ways (1) in a semi-field set-up where an Orius female could exploit many patches, and (2) by using results from observations on the foraging behaviour of the predator in a spatial simulation model.

The main objective of this work is to assess the number of $T$. palmi prey that an individual adult female $O$. sauteri consumes on average per day when foraging on eggplants. When $O$. sauteri females are used as a biological control agent in greenhouses, they visit numerous patches in 1 day. Thus, for one female, the foraging day consists of a sequence of patch visits. Because we have experimental data on the behaviour of one individual predator foraging for prey on one egg-plant leaf with different numbers of prey items present, we have a good starting point from which to scale up. Our earlier findings concerning the foraging process and the patch-leaving behaviour of $O$. sauteri are that, irrespective of the actual density of prey items on a patch, the leaving tendency only decreased by a factor of about 3 on average because prey were present (Yano et al. 2005). Moreover, the leaving tendency increases when patch exploitation lasts longer. To assess the number of $T$. palmi consumed per day by $O$. sauteri, we scale up from these earlier findings on how its foraging process and its patch-leaving behaviour are affected. We have developed a simulation model in which the simulated individual encounters a realistic distribution of prey over eggplant leaves (extracted from mean crowding estimations in Kawai 1986). This crowded distribution is simulated with a negative binomial distribution of prey over leaves, because insects are often found to have a clumped distribution (Harcourt 1961; Atkinson and Shorrocks 1984). The simulation model is first validated in two ways, and its results are discussed against the background of earlier experimental findings on a (simulated) functional response by Van Roermund et al. (1997).

\section{Methods}

The structure of the simulation model

The patch that is considered in the simulation model (see flowchart in Fig. 1) is the eggplant leaf. The simulation model is not spatially explicit. During the simulated day the predator visits prey patches, and every time that the O. sauteri individual leaves a patch, it reaches a randomly drawn new patch after the predator has travelled during a time span drawn from an exponential distribution. This new patch has a prey density (number per leaf) that is drawn from a clumped (i.e., negative binomial) distribution (for details on the distribution, see below). The decision process for the predator for whether it encounters and eats a prey item or whether it leaves before encountering a prey is modelled by drawing (1) a value of a giving-up time (GUT) based on the time the predator is already on the patch and whether prey are present or absent (see text and Eq. 1 below), and (2) the interval between encounters with prey (IBE) from an exponential distribution. On each leaf the predator encounters and eats a prey item (if drawn GUT $>$ drawn IBE) or leaves the patch (when drawn GUT $\leq$ drawn IBE), like in earlier simulation studies (Driessen and Hemerik 1992; Driessen and Bernstein 1999; Vos and Hemerik 2003; Wajnberg et. al. 2000). The handling time of prey items is also drawn from an exponential distribution in the simulation. The number of patches is not predetermined in the simulations, and by decreasing the travel times between patches one can take into account the fact that eggplants grow more leaves after having been planted. The simulations were performed in the $\mathrm{R}$ language version 2.8.0 (R Development Core Team 2008). 


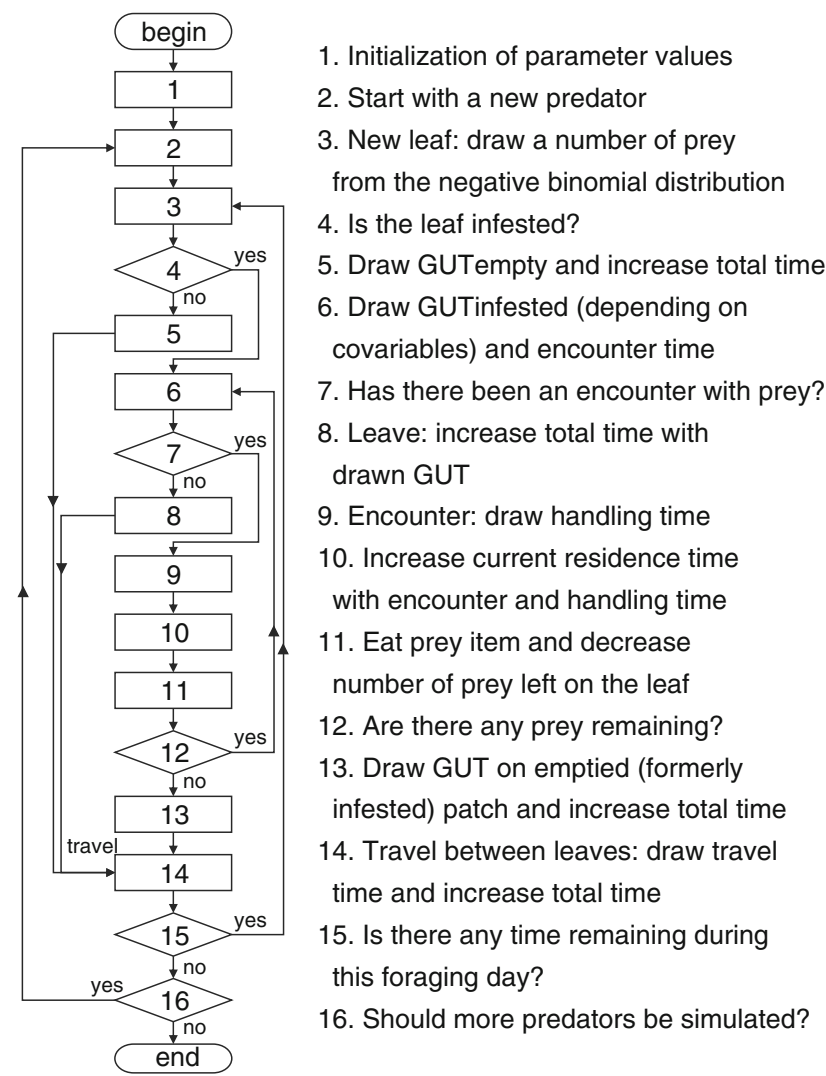

Fig. 1 Flowchart visualizing the decision process of the predatory bug $O$. sauteri; herewith its functional response during 1 day of foraging on eggplant leaves in a greenhouse is determined

\section{Parameter values}

As we want to determine the number of 2 nd instar larvae of $T$. palmi that one female adult of the species $O$. sauteri consumes in one foraging day, we have based our simulation model on studies where the patch-leaving behaviour was studied (Yano et al. 2005; see "Patch-leaving rate" and "Other rate parameters" below) and where the distribution of thrips larvae is determined in greenhouses (Kawai 1986; see "Parameterizing the clumped distribution of thrips over leaves" below).

The parameters that are used in the model are summarized in Table 1 together with their descriptions and estimated values. The rate parameters all describe exponentially distributed durations with mean equal to the reciprocal of the rate parameter value. All estimated parameter values were from data obtained at ambient temperature of approximately $25^{\circ} \mathrm{C}$, according to the estimated encounter rate and patch-leaving rate from the experiments reported in Yano et al. (2005).

Orius sauteri is active and forages only during daytime (K. Nagai, personal observation). For the simulation of 1 day we use the total average time in one foraging day of
O. sauteri in central Japan, where the mean day length in spring to autumn is $14 \mathrm{~h}$.

\section{Patch-leaving rate}

In Yano et al. (2005) a Cox proportional hazards model for the patch-leaving rate $h(t$, pres, time $)\left[\mathrm{s}^{-1}\right]$ was fitted to the censored and uncensored GUTs. Here, pres is coded as 0 or 1 when prey is absent from or present on the current patch, respectively (predators were not reacting to the actual prey density but they reacted upon presence versus absence of prey). The variable time is the time (in minutes) since the start of the current leaf visit at the latest renewal point. When looking closely at the estimated cumulative baseline hazard for the Cox proportional hazards model it became clear that its derivative, i.e., the baseline hazard, was approximately constant. Therefore we re-analysed the data with parametric survival models with the same renewal points of the patchleaving process as in Yano et al. (2005), namely entry to a patch and resuming the search after a successful encounter with a prey. The advantage of using a parametric model instead of the semi-parametric Cox proportional hazards model is that, in the parametric model, the baseline hazard is also estimated by one or more parameters. By comparing the outcomes of a Weibull model and an exponential model it became clear that the baseline leaving rate $h_{0}$ really was constant, because the scale parameter in the Weibull model was not significantly different from one. From the exponential model we thus got an estimate for $h_{0}$. The parameters $\beta_{\text {pres }}, \beta_{\text {time }}$ and $h_{0}$ (in Eq. 1) are the regression coefficients for pres and time and the baseline patch-leaving rate as estimated for the parametric survival model (1) that is fitted to the data from the behavioural experiments.

$h(t$, pres, time $)=h_{0} \exp \left(\beta_{\text {pres }}\right.$ pres $+\beta_{\text {time }}$ time $)$.

The parameter estimates for the $\beta\left(\beta_{\text {pres }}=-1.30\right.$, $\left.\beta_{\text {time }}=0.012, \quad h_{0}=1.143 \mathrm{E}-03\right) \quad$ are only slightly different from those reported as the estimates in the Cox proportional hazards model (Yano et al. 2005).

To be able to scale up from eggplant leaf level to greenhouse level, the distribution of the GUTs on empty patches as parameterized by the baseline hazard $h_{0}$ has to be known, otherwise the realizations of the stochastic process cannot be simulated. On empty patches the mean GUT is estimated as $875 \mathrm{~s}\left(=1 / h_{0}\right)$. The value for the regression parameter $\beta_{\text {pres }}$ indicates that the average GUT on patches with prey is $3.7[=\exp (1.30)]$ times larger than on empty patches. Both of these average times are influenced by the time (in minutes) since patch entry: each minute reduces the average time by a factor 0.988 $[=\exp (-0.012)]$. 
Table 1 Description of the parameters in the model

\begin{tabular}{|c|c|c|c|}
\hline Parameter & Description & Unit & Mean value \\
\hline$\beta_{\text {pres }}$ & $\begin{array}{l}\text { Regression coefficient of proportional hazards model giving the effect on the baseline hazard rate } \\
\text { when prey are present }\end{array}$ & - & -1.30 \\
\hline$\beta_{\text {time }}$ & $\begin{array}{l}\text { Regression coefficient of proportional hazards model giving the effect on the baseline hazard rate } \\
\text { per minute since patch entry at the latest encounter with prey }\end{array}$ & $\min ^{-1}$ & 0.012 \\
\hline$h_{0}$ & Baseline hazard rate on empty patch & $\mathrm{s}^{-1}$ & $1 / 875$ \\
\hline$r_{\mathrm{enc}}$ & Encounter rate with prey items & $\mathrm{s}^{-1}$ & $1 / 709$ \\
\hline$r_{\mathrm{h}}$ & Handling rate of prey items & $\mathrm{s}^{-1}$ & $1 / 200$ \\
\hline$r_{\text {travel }}$ & Travelling rate between patches & $\mathrm{s}^{-1}$ & $1 / 100$ \\
\hline$m$ & Mean of the negative binomial distribution of thrips over eggplant leaves & $\begin{array}{r}\text { number } \\
\text { leaf }^{-1}\end{array}$ & $\begin{array}{l}\text { Number of } \\
\text { values }^{\mathrm{a}}\end{array}$ \\
\hline$k$ & Aggregation parameter of the negative binomial distribution of thrips over eggplant leaves & - & $\begin{array}{l}\text { Number of } \\
\text { values }^{\mathrm{a}}\end{array}$ \\
\hline$T$ & Total foraging time in 1 day $(14 \mathrm{~h})$ & $\mathrm{s}$ & 50400 \\
\hline
\end{tabular}

a For relationships between $m$ and $k$ we refer to "Parameterizing the clumped distribution of thrips over leaves"

\section{Other rate parameters}

As stated above, both the handling time and the travel time are stochastic and are assumed to follow an exponential distribution. The average handling time of prey is $200 \mathrm{~s}$ (Yano et al. 2005), and the travelling time between patches is set at $100 \mathrm{~s}$ based on the data of walking speed $(=0.2967 \mathrm{~cm} / \mathrm{s})$, walking activity, i.e., the fraction of the total time that the bug is active $(=0.6933)$, and the mean distance between adjacent leaves $(=20 \mathrm{~cm})(\mathrm{K}$. Ohno and E. Yano, unpublished data). It was assumed that $O$. sauter individuals travel by walking between patches based on observation of patch-leaving behaviour (E. Yano, personal observation).

For the encounter rate of prey items, the same approach is used as in the appendix of Vos and Hemerik (2003). Those authors assume that both the IBEs and the GUTs result from an exponential distribution with parameters $r_{\mathrm{enc}}$ and $h_{0}$, respectively. From our data set on $O$. sauteri foraging on $T$. palmi, we calculated the overall mean $g$ for all observed IBEs and GUTs together on prey-infested patches. This equals $g=1 /\left(r_{\text {enc }}+h_{0}\right)$. The calculated value was $539 \mathrm{~s}$, and the number of realized IBE intervals and GUT intervals in our data were 162 and 52 (fractions 0.76 and 0.24 ). Under the assumption of exponentially distributed encounters and GUTs, we know from the appendix of Vos and Hemerik (2003) that the fraction of realized encounters with prey $\left(f_{\text {enc }}\right)$ and the fraction of realized GUTs $\left(1-f_{\text {enc }}\right)$ are derived as, respectively, $f_{\text {enc }}=r_{\text {enc }} l$ $\left(r_{\text {enc }}+h_{0}\right)$ and $1-f_{\text {enc }}=h_{0} /\left(r_{\text {enc }}+h_{0}\right)$. Therefore, the mean duration of IBE and GUT in all experiments are, respectively, $709 \mathrm{~s}\left(=g / f_{\text {enc }}\right)$ and $2246 \mathrm{~s}\left(=g /\left(1-f_{\text {enc }}\right)\right)$. In the simulation we only use the mean value for the IBEs explicitly $\left[r_{\text {enc }}=(1 / 709) \mathrm{s}^{-1}\right.$, see Table 1], because the other estimate is already simulated with the leaving rate and the effects ( $\beta_{\text {pres }}$ and $\left.\beta_{\text {time }}\right)$ as estimated with the parameterized survival model given in Eq. 1 .

\section{Parameterizing the clumped distribution of thrips over leaves}

Many insect species have a negative binomial distribution over patches (Harcourt 1961; Atkinson and Shorrocks 1984). For convenience, in Eq. 2 we give the formula for finding a patch with $i$ prey items when the number of prey items follows a negative binomial distribution over patches. The mean of the negative binomial distribution is $m$ prey items, $k$ is the clumping parameter and its variance is $m+\frac{m^{2}}{k}$ (Southwood and Henderson 2000).

$P(X=i)=\left(\begin{array}{c}k+i-1 \\ i\end{array}\right)\left(\frac{k}{m+k}\right)^{k}\left(\frac{m}{m+k}\right)^{i}$.

For T. palmi, Kawai (1986) estimated the functional relationship between the mean crowding $m^{*}$ and the mean $m$ (number of thrip larvae per leaf). He did not compute the clumping parameter of the negative binomial distribution explicitly. Therefore, we have to look into the details of his estimated relationship: The mean crowding $m^{*}$ is defined as the mean number of neighbouring individuals per individual per quadrate. Its relation to the mean $m$ and variance $\sigma^{2}$ is known to be $m^{*}=m-1+\left(\sigma^{2} / m\right)$ (formula a from Iwao and Kuno 1968). In general, the relation between the mean and the mean crowding can be described with a simple linear regression as $m^{*}=\alpha+\beta m$ (formula b from Iwao 1977). For larvae of the species T. palmi, Kawai (1986) reported two different estimates for $\alpha$ and $\beta$ in such a linear regression equation, namely 
$(\alpha, \beta)=(4.11,1.34)$ or $(4.84,3.54)$, respectively. The former estimates were obtained from the survey in a greenhouse where the eggplants were just planted, and the latter estimates were for a later cropping period of eggplants. Combining regression values $\alpha$ and $\beta$ with the two formulas $\mathbf{a}$ and $\mathbf{b}$ for mean crowding and the mean and variance of the negative binomial distribution (just above Eq. 2) results in two different relationships between $k$ and $m$, namely $k=f_{1}(m)=m /(4.11+0.34 m)$ and $k=f_{2}(m)=m /(4.84+2.54 m)$. For our simulation study we used these two different functions to describe the relationship between the mean $m$ and the clumping parameter $k$ to be able to simulate numbers of prey items in a patch as a random draw from the negative binomial distribution with mean $m$ and clumping parameter $k$.

\section{Model validation}

Ideally, the model could be compared with data for one predator that could explore a semi-field set-up with, say, eight eggplant plants during $14 \mathrm{~h}$. Such data are not available, thus we have only tested the prey consumption and the patch-leaving part of our model. Additional data were collected on the number of prey eaten and the patch residence time for patches with 20 prey items as initial density (N. Jiang and E. Yano, unpublished data) in a setup as described in Yano et al. (2005). There were 24 replicates. It should be noted that this data set (used for testing the model) has been obtained independently from the data used by Yano et al. (2005). To test our model, we simulated the numbers eaten and the patch residence times on 1000 patches with initial prey density set to 20 . We compared the data and the simulation results for the number of prey eaten using a non-parametric Wilcoxon rank-sum test (for all 1000 simulations and for the same simulations divided into 40 subsets of 25 patches). For the patch residence times a Kaplan-Meier survivor plot was drawn and the samples were compared with a log-rank test (Klein and Moeschberger 1997). All analyses were done in the $R$ language version 2.8.0 (R Development Core Team 2008).

\section{Simulation scenarios}

We have performed simulations of the searching behaviour of $O$. sauteri at densities below or around the economic injury level of 0.55 second instar larvae of T. palmi per eggplant leaf to estimate the number of prey eaten by this predator during one foraging day. We also included mean densities of 1,2 or 3 per leaf, which are far above the normal levels encountered in a greenhouse, to acquire knowledge on how much one adult Orius predator is able to eat at such high densities in case one wants to start biological control after having measured these mean number of thrips per eggplant leaf. For each combination of $m$ and $k, 1000$ individual predators were simulated, essentially having access to an unlimited number of leaves with and without prey, because the number of prey on each leaf was drawn from a negative binomial distribution (which is clumped and therefore represents an over-representation of empty patches).

Statistical methods

For the different values of $k$ of the negative binomial distribution we tested with the Wilcoxon rank-sum test at each mean number of thrips per leaf whether there was a difference between the differently clumped distributions. These analyses were done in the $\mathrm{R}$ language version 2.8.0 (R Development Core Team 2008).

We also fitted three different Holling-type functions ((I) $y=a_{1} m$, (II) $y=\frac{a_{2} m}{b_{2}+m}$ and (III) $y=\frac{a_{3} m^{2}}{b_{3}^{2}+m^{2}}$, where $y$ is the number of prey eaten and $m$ is the mean prey density) to the functional responses and made a selection between these models using Akaike's information criterion (AIC, Akaike 1974). The model with minimum AIC is the best (the analysis was performed in the $\mathrm{R}$ language version 2.8.0 by non-linear regression).

\section{Sensitivity analysis}

We are quite sure that the parameter values that describe the searching and decision process of the predator are correctly estimated from our data (with low variance). However, we would still like to know how the parameters included in the model affect the functional response of $O$. sauteri. Therefore, we performed a sensitivity analysis on each of the 6 following parameters: $h_{0}, r_{\mathrm{enc}}, r_{\mathrm{h}}, r_{\text {travel }}$, $\beta_{\text {pres }}$ and $\beta_{\text {time. }}$. To get a good view of whether parameter changes cause large variations we varied the parameter values by $-50 \%$ and $+50 \%$ at mean prey densities of $m=0.1,0.3,0.5,1$ or 2 prey per leaf with their corresponding $k$ values of the more clumped distribution described by the relation $k=f_{2}(m)=m /(4.84+2.54 m)$.

\section{Results}

Model validation

We compared the simulated numbers of prey eaten in 25 patch visits on patches with 20 prey items as initial density $(n=1000$, overall mean number $=2.97)$ with the observed numbers of prey eaten $(n=24$, mean $=3.46)$. The observations (Fig. 2a) can easily be compared with the simulations in the histograms provided in Fig. $2 b-$ d. The 
Fig. 2 Histograms for the realized number of prey eaten in (a) the 24 observed patches. The experiments in a were performed at $25^{\circ} \mathrm{C}$. b-d Three realizations from a stochastic process, namely the first three simulated sets of 25 patches. In both the observations and the simulations the initial number of prey in the patches was 20
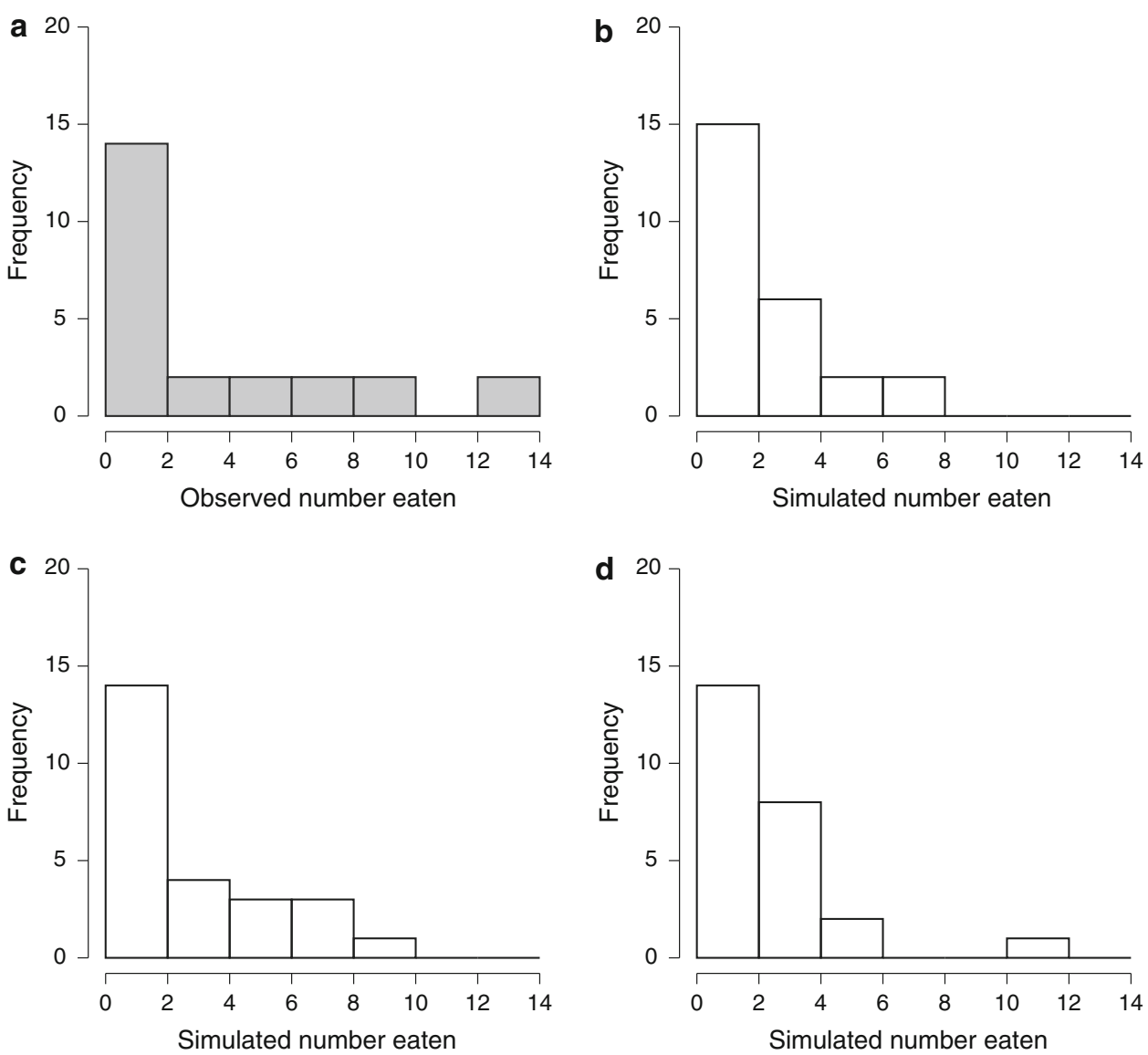

observed data of the cumulative number of prey eaten in one patch with 20 prey items were not significantly different from the simulated data [two-sample $t$ test with median $P$ value in the comparisons of 0.65 (with interquartile distance 0.46-0.84)]. For the patch residence times, the survivor functions are given in Fig. 3 and the log-rank test (testing the null hypothesis of no difference between observations and simulations) showed no indication of significant difference $(P=0.481)$. The average patch residence time in the simulation was $2742 \mathrm{~s}$ and in the observations $2727 \mathrm{~s}$.

\section{Simulation}

It should be noted that the different relations between mean $m$ and clumping parameter $k$ represent more clumped distributions when the $k$ value is smaller at the same mean value $m$. Therefore, the second relationship between $k$ and $m$, i.e., $k=f_{2}(m)$, represents the more clumped distribution. The number of prey that one predator can eat in 1 day at densities around the economic injury level $(m=0.45-0.65$ per leaf) is on average 10 (range 1-32) for both $k$ series. The more clumped distribution results in a somewhat lower mean number of prey eaten per day (Fig. 4a) at high mean prey densities (Wilcoxon rank-sum test: $P \ll 0.001$ for

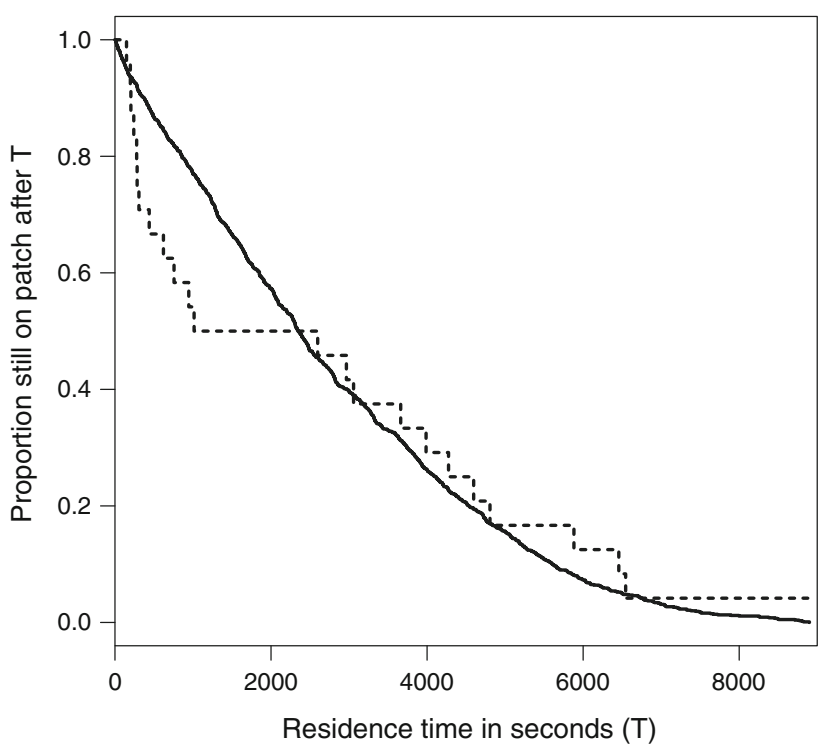

Fig. 3 Kaplan-Meier survivor plot for the simulated patch residence times (solid line, $n=1000$ ) and the observed patch residence times (dashed line, $n=24$ )

mean density 2 and 3), but at low prey densities the results do not differ. This mainly represents the fact that a more clumped distribution is a distribution in which some patches have high prey density, but there are also relatively more 

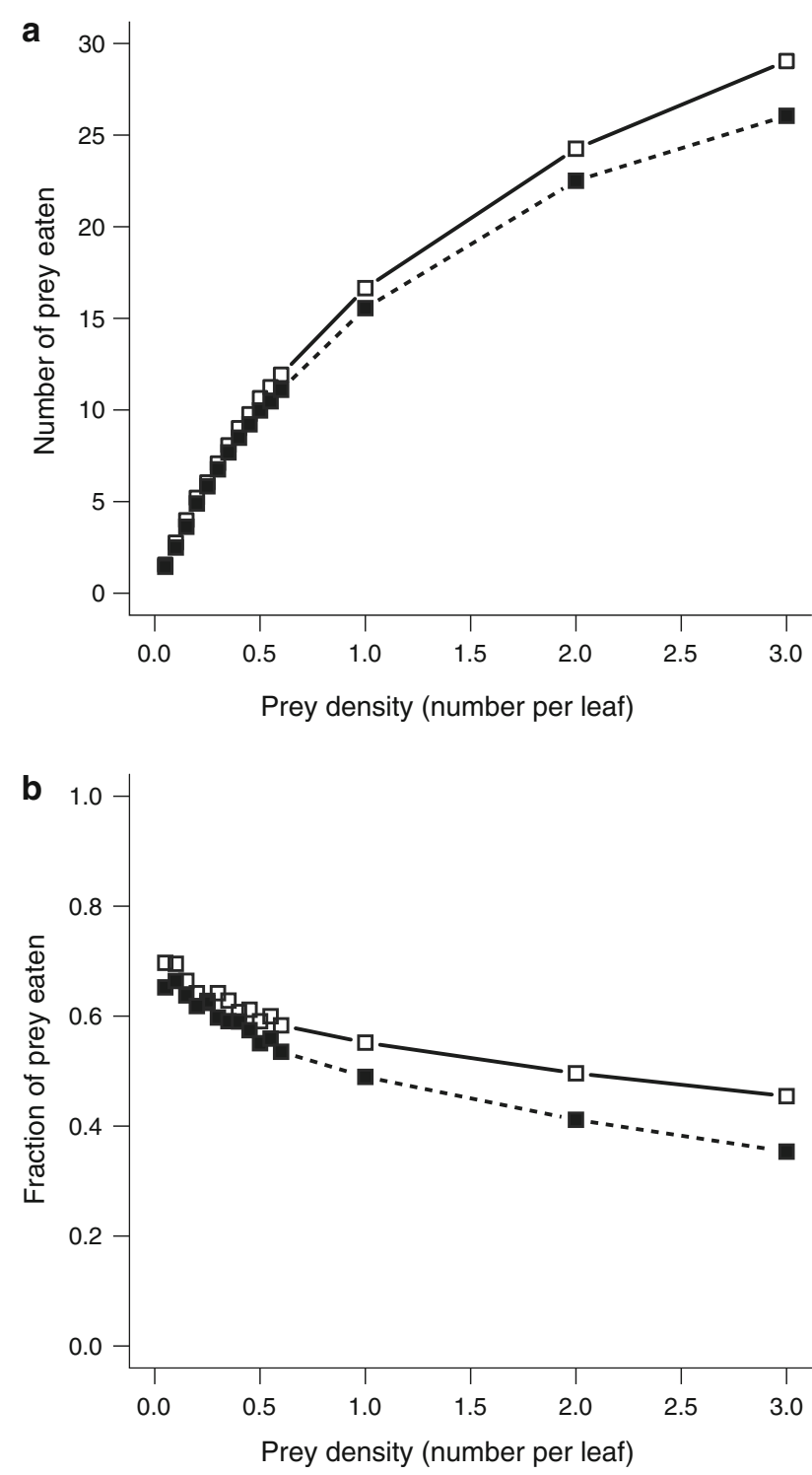

Fig. 4 Results of the simulations with a negative binomial distribution of prey over patches with mean density $m$ number per leaf on the $x$-axis and clumping parameter $k$ denoted by open squares [for relationship $k=f_{1}(m)=m /(4.11+0.34 m)$ ] and filled squares [for relationship $k=f_{2}(m)=m /(4.84+2.54 m)$ ]. a The number of prey consumed on average by 1000 simulated $O$. sauteri in one foraging day. b The average fraction of the host on the visited patches that is consumed per day by one $O$. sauteri bug

empty patches to find. The number of consumed prey is lower in the more clumped distribution because relatively more time is wasted on empty patches. When fitting the three Holling-type functional response relationships as stated in the "Statistical methods" to the simulation results, the Holling type II functional response had for both $k$ series a negative AIC value $\left(\mathrm{AIC}_{\mathrm{II}-f_{1}}=-1.37, \quad \mathrm{AIC}_{\mathrm{II} \_} f_{2}=\right.$ -10.92) while the linear $\left(\mathrm{AIC}_{\mathrm{I} \_} f_{1}=87.15, \mathrm{AIC}_{\mathrm{I} \_} f_{2}=\right.$ 86.45) and sigmoid relationship ( $\mathrm{AIC}_{\mathrm{III} \_} f_{1}=66.85$,
$\mathrm{AIC}_{\mathrm{III}} f_{2}=63.34$ ) had positive AIC values. Thus the Holling type II functional response was the best fitting model for both $k$ series. The consumed fraction of the total prey population that was present on all patches together during the full foraging day was decreasing with the mean prey density $m$ per leaf (Fig. 4b). At mean values 2 and 3 the difference was significant, for each Wilcoxon rank-sum test $(P \ll 0.001)$. For both $k$ series the fraction eaten at mean densities around the economic injury level was approximately $55-60 \%$.

\section{Sensitivity analysis}

For the most clumped $k$ series we changed the values of the six parameters $h_{0}, r_{\text {enc }}, r_{\mathrm{h}}, r_{\text {travel }}, \beta_{\text {pres }}$ and $\beta_{\text {time }}$ by $-50 \%$ or $+50 \%$. Varying the parameter $\beta_{\text {time }}$, which increases the leaving tendency during the course of a patch visit, only caused a change in the number of prey eaten in the range of $-3 \%$ to $+3 \%$ at the five investigated mean prey densities. The handling time modelled as the rate $r_{\mathrm{h}}$ affected the fraction of prey eaten by differences in the range from $-10 \%$ to $+1 \%$. The number of prey eaten when changing the travel times as given by the rate $r_{\text {travel }}$ varied between $-9 \%$ and $+7 \%$. Thus, these parameters did not have a great effect on the number of prey eaten. Table 2 shows the results of how changes in the baseline leaving tendency $h_{0}$, the prey encounter rate $r_{\mathrm{enc}}$ and the behavioural parameter that makes an individual search longer in a prey-infested patch $\beta_{\text {pres }}$ affect the mean number of prey eaten by one individual $O$. sauteri predator. All three of these parameters influenced the resulting number of prey eaten to a greater extent (ranges $h_{0}-30 \%$ to $+24 \%, r_{\mathrm{enc}}-34 \%$ to $+23 \%, \beta_{\text {pres }}-19 \%$ to $\left.+25 \%\right)$ than the formerly reported parameters. Figure $5 \mathrm{a}-\mathrm{c}$ shows how the resulting number of prey eaten per day by one $O$. sauteri depends on the value of $h_{0}, r_{\mathrm{enc}}$ and $\beta_{\text {pres }}$ in our simulations.

How the parameter values influence the fraction eaten by one individual $O$. sauteri predator of the total number of prey encountered in the visited patches is shown in Table 3, for the three parameters with the greatest influence: the baseline leaving tendency $h_{0}$, the prey encounter rate $r_{\text {enc }}$ and the behavioural parameter that makes an individual search longer in a prey-infested patch $\beta_{\text {pres }}$ (ranging from $-20 \%$ to $+40 \%$, from $-34 \%$ to $+22 \%$ and from $-32 \%$ to $+37 \%$, respectively). Varying the parameter $\beta_{\text {time }}$ only caused a change in the range of $-5 \%$ to $+8 \%$ at the five investigated mean prey densities. The handling time modelled as the rate $r_{\mathrm{h}}$ and the travel times as given by the rate $r_{\text {travel }}$ affected the fraction of prey eaten only slightly (ranging from $-4 \%$ to $+4 \%$ ). As an illustration, Fig. $5 \mathrm{~d}$ show the dependence of the fraction of prey eaten on the encounter rate. When the absolute effect size of $\beta_{\text {pres }}$ is higher, the fraction of encountered prey that are eaten 
Fig. 5 Example results of sensitivity analysis for the series with mean density $m$ and $k$ values from the more clumped distribution: a influence of the baseline leaving tendency $h_{0}$, b influence of the encounter rate $r_{\text {enc }}, \mathbf{c}$ influence of the presence of prey $\beta_{\text {pres }}$ on the number of prey eaten and $\mathbf{d}$ influence of the encounter rate $r_{\mathrm{enc}}$ on the fraction of the total host population eaten in the visited patched during 1 day. a-d Low (triangles), medium (squares) and high (circles) values of the considered model parameter mean that its value is set, respectively, to $0.5,1$ and 1.5 times the default value
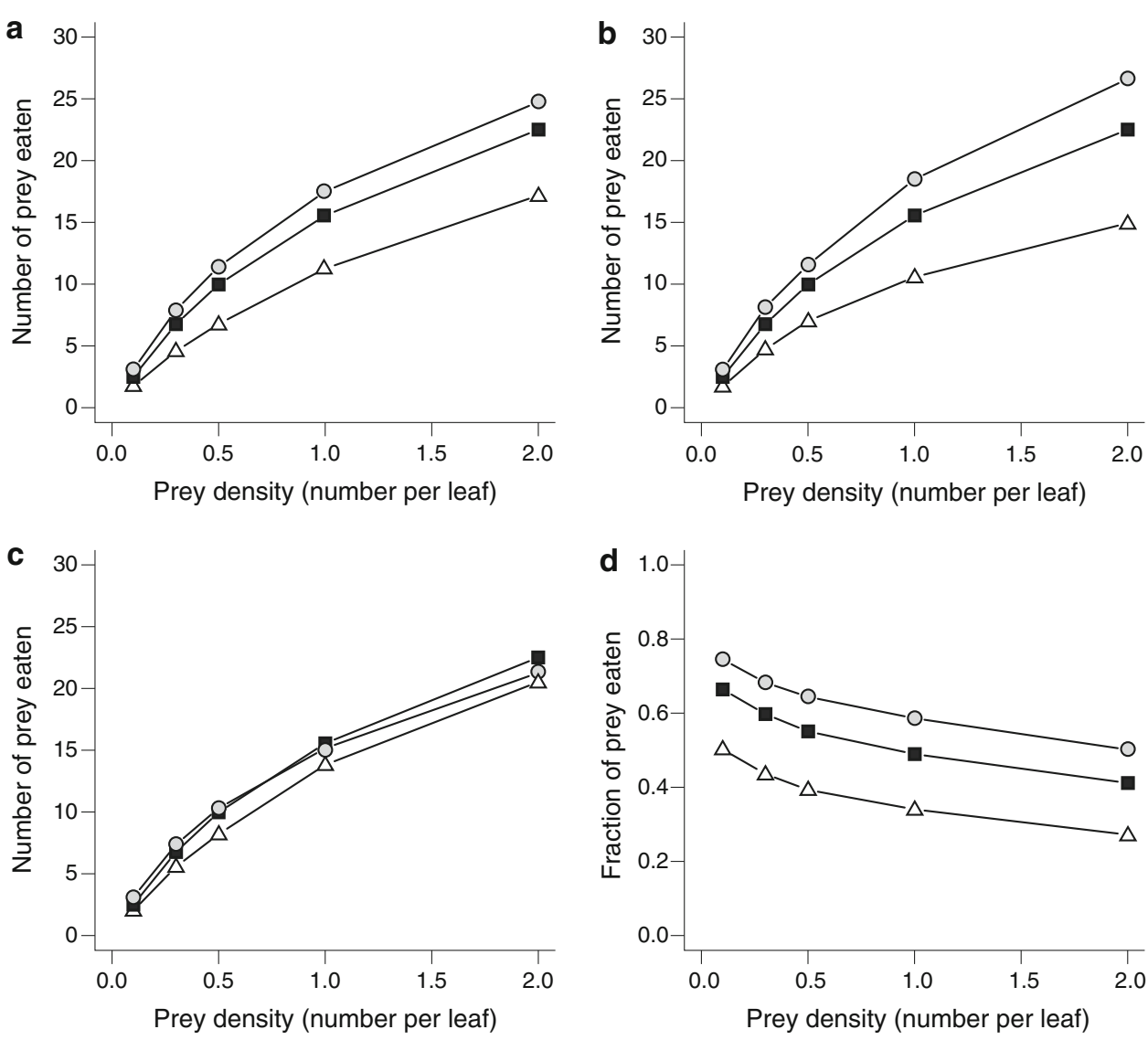

Table 2 Results of sensitivity analysis for the number of prey eaten in one foraging day

\begin{tabular}{|c|c|c|c|c|c|c|}
\hline & \multicolumn{2}{|l|}{$h_{0}$} & \multicolumn{2}{|l|}{$r_{\mathrm{enc}}$} & \multicolumn{2}{|l|}{$\beta_{\text {pres }}$} \\
\hline & $-50 \%$ & $+50 \%$ & $-50 \%$ & $+50 \%$ & $-50 \%$ & $+50 \%$ \\
\hline$m=0.1$ & $-30 \%$ & $+24 \%$ & $-31 \%$ & $+23 \%$ & $-19 \%$ & $+25 \%$ \\
\hline$m=0.3$ & $-32 \%$ & $+17 \%$ & $-30 \%$ & $+20 \%$ & $-18 \%$ & $+9 \%$ \\
\hline$m=0.5$ & $-32 \%$ & $+15 \%$ & $-30 \%$ & $+16 \%$ & $-18 \%$ & $+3 \%$ \\
\hline$m=1$ & $-28 \%$ & $+13 \%$ & $-32 \%$ & $+19 \%$ & $-11 \%$ & $-3 \%$ \\
\hline$m=2$ & $-24 \%$ & $+10 \%$ & $-34 \%$ & $+18 \%$ & $-9 \%$ & $-6 \%$ \\
\hline
\end{tabular}

Values are maximum/minimum percentage changes in the response variable for each parameter varied

increases because the predator is more persistent in searching for prey on prey-infested leaves.

\section{Discussion}

First we take a critical look at the assumptions of the model. As we have pointed out above, our simulation model is not spatially explicit. Therefore, we have not included a preference for detecting prey-infested patches, although it is known that predatory bugs of the genus Orius
Table 3 Results of sensitivity analysis for the fraction eaten of the total number of prey encountered in one foraging day

\begin{tabular}{|c|c|c|c|c|c|c|}
\hline & \multicolumn{2}{|l|}{$h_{0}$} & \multicolumn{2}{|l|}{$r_{\mathrm{enc}}$} & \multicolumn{2}{|l|}{$\beta_{\text {pres }}$} \\
\hline & $-50 \%$ & $+50 \%$ & $-50 \%$ & $+50 \%$ & $-50 \%$ & $+50 \%$ \\
\hline$m=0.1$ & $+19 \%$ & $-13 \%$ & $-24 \%$ & $+12 \%$ & $-25 \%$ & $+18 \%$ \\
\hline$m=0.3$ & $+28 \%$ & $-19 \%$ & $-27 \%$ & $+14 \%$ & $-25 \%$ & $+22 \%$ \\
\hline$m=0.5$ & $+35 \%$ & $-17 \%$ & $-29 \%$ & $+17 \%$ & $-27 \%$ & $+28 \%$ \\
\hline$m=1$ & $+36 \%$ & $-17 \%$ & $-30 \%$ & $+20 \%$ & $-28 \%$ & $+30 \%$ \\
\hline$m=2$ & $+40 \%$ & $-20 \%$ & $-34 \%$ & $+22 \%$ & $-32 \%$ & $+37 \%$ \\
\hline
\end{tabular}

Values are maximum/minimum percentage changes in the response variable for each parameter varied

are preferentially attracted to herbivore-infested patches (Venzon et al. 1999; Mochizuki and Yano 2007). It is also not quantitatively known whether and to what extent arrival tendencies to prey-infested plants differ. Including such a preference when choosing between neighbouring patches would have increased the predation capability slightly, because a clumped distribution of hosts implies also a lot of empty patches that are neighbouring each other. The exponentially distributed handling times and arrival times represent the best and simplest distributions for such variable times that are not quantified in detail. 
The simulation results of the model depicted in Fig. 1 show that one female adult of $O$. sauteri can find and eat approximately 10 prey individuals per day at a level of infestation around the economic injury level of T. palmi (an average of 0.55 individuals per leaf). Since the intrinsic rate of natural increase of $T$. palmi at $25^{\circ} \mathrm{C}$ is estimated at 0.102 per day (Kawai 1986), it is calculated that the T. palmi population increases only by approximately $11 \%$ per day. For a not too small ratio of the number of $O$. sauteri to $T$. palmi, $O$ sauteri has great potential to suppress the T. palmi population. Since the functional response is the individual predator response to the prey density, we have not included interference or competition between predators, because no visual response was observed when two individuals met each other. When predators compete with each other, this might reduce the number of prey eaten per day per individual. However, densities of the predator in biological control are generally not so high; for example, in the study by Kawai (1995), the maximum density of the total of nymphs and adults of O. sauteri per eggplant leaf in five greenhouses was 0.21 in about 60 days after 0.5 or 1.0 nymphs per leaf were released once in each greenhouse.

Most experimental studies in very small homogeneous spaces such as a Petri dish or a plastic vial have shown a type II functional response by Orius spp. to densities of different types of prey (McCaffrey and Horsburgh 1986; Isenhour et al. 1989, 1990; Coll and Ridgway 1995; Nagai and Yano 2000; Gitonga et al. 2002). Among them, Nagai and Yano (2000) studied the functional response of $O$. sauteri to densities of T. palmi and found at $25^{\circ} \mathrm{C}$ a type II response for a female adult for aging at different densities of the second instar larvae. The current simulation study also results in a type II response of $O$. sauteri foraging at low densities of $T$. palmi around its economic injury level. It was also shown that the distribution of T. palmi among patches has a great influence on the shape of the functional response (a homogeneous distribution leads to a type I functional response). This kind of effect of the distribution of prey on the functional response is not found often. We know of one occasion where in a simulation study the distribution of hosts had a similar effect: When modelling the foraging behaviour of Encarsia formosa Gahan (Hymenoptera, Aphelinidae) attacking immature greenhouse whitefly Trialeurodes vaporariorum (Westwood) (Homoptera, Aleyrodidae), functional responses of E. formosa to immature whitefly densities were greatly affected by host distribution over leaflets, as those of $O$. sauteri to densities of thrip larvae (Van Roermund and Van Lenteren 1994; Van Roermund et al. 1997).

The results of the model validation strengthen our opinion that the details of the simulation model resemble the behaviour of $O$. sauteri foraging on eggplant leaves at ambient temperature of $25^{\circ} \mathrm{C}$ as explained at the end of the "Introduction". In addition to this fact, it is no surprise that the model outcomes are highly sensitive to the values of the baseline patch-leaving rate $h_{0}$ and the encounter rate $r_{\text {enc }}$. When the encounter rate is higher at the same baseline patch-leaving rate, more prey can be encountered and both the number of prey eaten and the fraction of the total prey population on the visited patches increase. If, alternatively, the baseline patch-leaving rate is higher at the same value of the encounter rate, then a patch is left earlier. Thus, the number of patches that are visited in the same amount of time and the total number of prey eaten both increase, but the consumed fraction of the total prey population on the visited patches decreases. How the number of prey eaten is influenced by the value of $\beta_{\text {pres }}$ is not so clear. The encounter rate with prey is only defined on prey-infested patches, and the behavioural parameter $\beta_{\text {pres }}$ influences the leaving tendency on such patches. Therefore, the resulting effect on the number of prey eaten in 1 day after having visited prey-infested and empty patches is not straightforward. This is also obscured by the fact that, in clumped distributions, there are some patches with high prey densities but also a lot of empty patches. The consumed fraction of prey is higher when $\beta_{\text {pres }}$ is higher because an increase in the negative value by $50 \%$ means that initially the GUT on patches with prey is multiplied by seven $[=\exp (1.5 \times 1.3)]$. For low mean prey densities this results in higher numbers eaten per day, but for high mean prey densities more time is wasted after some time on an eggplant leaf, because the predator prolongs its GUT on preyinfested patches.

In the simulation study of Van Roermund et al. (1997) at high host densities with a clustered distribution of hosts over leaflets, the GUT on a leaflet was the most essential parameter influencing the parasitoid-host dynamics. Outcomes of our study are also highly sensitive to the value of the baseline patch-leaving rate $h_{0}$. Other parameters could not be compared with the results of Van Roermund et al. (1997) because of differences in model structures and in foraging behaviour between E. formosa and O. sauteri.

With this simulation study we have quantified the daily value of the functional response for a general predatory anthocorid bug, suggesting that this value allows for effective control of thrips by such a predator. The previously performed behavioural analysis (Yano et al. 2005) served as a starting point for the quantification of the impact that one individual $O$. sauteri has on its prey population at ambient temperature of $25^{\circ} \mathrm{C}$. If the predatorprey dynamics of $O$. sauteri on $T$. palmi are investigated in future studies, the most appropriate functional response to use is Holling type II. 
Acknowledgment Most of this work was performed in Japan, during L.H.'s stay that was subsidized by the Japanese Society for the Promotion of Science (JSPS ID S-08116). We thank Wopke Van Der Werf and Herman Van Roermund for constructive comments upon an earlier version of the manuscript, and Elizabeth Van Ast for correction of the English.

Open Access This article is distributed under the terms of the Creative Commons Attribution Noncommercial License which permits any noncommercial use, distribution, and reproduction in any medium, provided the original author(s) and source are credited.

\section{References}

Akaike H (1974) A new look at the statistical model identification. IEEE Trans Autom Contr 19:716-723

Atkinson WD, Shorrocks B (1984) Aggregation of larval diptera over discrete and ephemeral breeding sites: the implications for coexistence. Am Nat 124:336-351

Birch LC (1948) The intrinsic rate of natural increase of an insect population. J Anim Ecol 17:15-26

Coll M, Ridgway RL (1995) Functional and numerical responses of Orius insidiosus (Heteroptera: Anthocoridae) to its prey in different vegetable crops. Ann Entomol Soc Am 88:732-738

Driessen G, Bernstein C (1999) Patch departure mechanisms and optimal host exploitation in an insect parasitoid. J Anim Ecol 68:445-459

Driessen G, Hemerik L (1992) The time and egg budget of Leptopilina clavipes, a parasitoid of larval Drosophila. Ecol Entomol 17:17-27

Gitonga LM, Overholt WA, Löhr B, Magambo JK, Mueke JM (2002) Functional response of Orius albidipennis (Hemiptera: Anthocoridae) to Megalurothrips sjostedti (Thysanoptera: Thripidae). Biol Control 24:1-6

Harcourt DG (1961) Spatial pattern of the imported cabbageworm, Pieris rapae (L.) (Lepidoptera: Pieridae), on cultivated Cruciferae. Can Entomol 93:945-952

Holling CS (1959) Some characteristics of simple types of predation and parasitism. Can Entomol 91:385-398

Isenhour DJ, Wiseman BR, Layton RC (1989) Enhanced predation by Orius insidiosus (Hemiptera: Anthocoridae) on larvae of Heliothis zea and Spodoptera frugiperda (Lepidoptera: Noctuidae) caused by prey feeding on resistant corn genotypes. Environ Entomol 18:418-422

Isenhour DJ, Layton RC, Wiseman BR (1990) Potential of adult Orius insidiosus (Hemiptera: Anthocoridae) as a predator of the fall armyworm, Spodoptera frugiperda (Lepidoptera: Noctuidae). Entomophaga 35:269-275

Iwao $S$ (1977) The $m^{*}-m$ statistics as a comprehensive method for analyzing spatial patterns of biological populations and its application to sampling problems. In: Morisita M (ed) Studies on methods of estimating population density, biomass and productivity in terrestrial animals. Japanese Committee for the International Biological Program. University of Tokyo Press, Tokyo, pp 21-46

Iwao S, Kuno E (1968) Use of the regression of mean crowding on mean density for estimating sample size and the transformation of data for the analysis of variance. Res Popul Ecol 10:210-214

Kawai A (1986) Studies on population ecology and population management of Thrips palmi Karny. Bull Veg Orn Crops Res Stn 9:69-135 (in Japanese with English summary)
Kawai A (1995) Control of Thrips palmi Karny (Thysanoptera: Thripidae) by Orius spp. (Heteroptera: Anthocoridae) on greenhouse eggplant. Appl Entomol Zool 30:1-7

Klein JP, Moeschberger ML (1997) Survival analysis: techniques for censored and truncated data. Springer, New York

McCaffrey RL, Horsburgh JP (1986) Functional response of Orius insidiosus (Hemiptera: Anthocoridae) to the European red mite, Panonychus ulmi (Acari: Tetranychidae), at different constant temperatures. Environ Entomol 15:532-535

Mochizuki M, Yano E (2007) Olfactory response of the anthocorid predatory bug Orius sauteri to thrips-infested eggplants. Entomol Exp Appl 123:57-62

Nagai K (1993) Studies on integrated pest management of Thrips palmi Karny. Spec Bull Okayama Pref Agric Exp Stn 82:1-55 (in Japanese with English summary)

Nagai K, Yano E (1999) Effects of temperature on the development and reproduction of Orius sauteri (Poppius) (Heteroptera: Anthocoridae), a predator of Thrips palmi Karny (Thysanoptera: Thripidae). Jpn J Appl Entomol Zool 34:223-229

Nagai K, Yano E (2000) Predation by Orius sauteri (Poppius) (Heteroptera: Anthocoridae) on Thrips palmi Karny (Thysanoptera: Thripidae): functional response and selective predation. Jpn J Appl Entomol Zool 35:565-574

Ohno K, Takemoto H (1997) Species composition and seasonal occurrence of Orius spp. (Heteroptera: Anthocoridae), predacious natural enemies of Thrips palmi (Thysanoptera: Thripidae), in eggplant fields and surrounding habitats. Jpn J Appl Entomol Zool 32:27-35

R Development Core Team (2008) R: a language and environment for statistical computing. R Foundation for Statistical Computing, Vienna, Austria. ISBN: 3-900051-07-0. http://www.R-project.org

Southwood TRE, Henderson PA (2000) Ecological methods. Blackwell, Oxford

Takemoto H, Ohno K (1996) Integrated pest management of Thrips palmi in eggplant fields, with conservation of natural enemies: Effects of the surroundings and thrips community on the colonization of Orius spp. In: Hokyo N, Norton G (eds) Pest management strategies in Asian monsoon agro-ecosystems. Kyushu Nat Agric Exp Stat, Kumamoto, pp 235-244

Van Roermund HJW, Van Lenteren JC (1994) The functional response of the parasitoid Encarsia formosa, searching for whitefly on a plant. Proc Neth Entomol Soc Meet 5:165-172

Van Roermund HJW, Van Lenteren JC, Rabbinge R (1997) Analysis of foraging behaviour on the whitefly parasitoid Encarsia formosa on a plant: a simulation study. Biocontrol Sci Technol 7:131-151

Venzon M, Janssen A, Sabelis MW (1999) Attraction of a generalist predator towards herbivore-infested plants. Entomol Exp Appl 93:305-314

Vos M, Hemerik L (2003) Linking foraging behavior to lifetime reproductive success for an insect parasitoid: adaptation to host distributions. Behav Ecol 14:236-245

Wajnberg E, Fauvergue X, Pons O (2000) Patch leaving decision rules and the marginal value theorem: an experimental analysis and a simulation model. Behav Ecol 11:577-586

Yano E (1996) Biology of Orius sauteri (Poppius) and its potential as a biocontrol agent for Thrips palmi Karny. IOBC WPRS Bull 19:203-206

Yano E, Jiang N, Hemerik L, Mochizuki M, Mitsunaga T, Shimoda T (2005) Time allocation of Orius sauteri in attacking Thrips palmi on an eggplant leaf. Entomol Exp Appl 117:177-184 